\title{
Non-invasive pre-clinical MR imaging of prostate tumor hypoxia for radiation therapy prognosis
}

\author{
Derek A White', Ralph P Mason² \\ ${ }^{I}$ UT Arlington Biomedical Engineering, Texas, USA. \\ ${ }^{2}$ UT Southwestern Prognostic Radiology Lab, Texas, USA.
}

Received March 19, 2014; Published Online April 08, 2014

\begin{abstract}
[Presented at the Young Investigator's Symposium at the 2014 Annual Meeting of Southwest Chapter of American Association of Physicists in Medicine (AAPM) in San Antonio, Texas, USA]
\end{abstract}

\section{Conference Proceeding}

\begin{abstract}
Purpose: To investigate the usefulness of Oxygen-Enhanced Magnetic Resonance Imaging (OE-MRI) changes in signal intensity related to oxygen challenge for predicting tumor response to radiation therapy.
\end{abstract}

Methods: Dynamic MR signal changes were acquired using Varian 4.7T small animal MR scanner prior to image-guided radiation therapy (IGRT) of small $(n=6)$ and large subcutaneous $(n=5)$ prostate tumors in adult male rats. An interleaved blood-oxygen level dependent (BOLD) and tissue-oxygen level dependent (TOLD) data acquisition or (IBT) was performed using a baseline of medical air as positive control and using medical oxygen as a breathing challenge. BOLD used a 2-D multi-slice spoiled gradient-echo with multi-echo sequence. TOLD used a 2-D multi-slice spoiled gradient-echo sequence. Voxel changes in signal intensity were determined by a correlation coefficient mapping technique. Irradiation technique planned consisted of $1 \mathrm{~F} \times 15 \mathrm{~Gy}$ $\mathrm{AP} / \mathrm{PA}$ or $2 \mathrm{~F} \times 7.5 \mathrm{~Gy} \mathrm{AP} / \mathrm{PA}$ to the gross tumor volume (GTV). Tumor growth measurements were recorded over time to assess the response to IGRT.

Results: BOLD and TOLD signals variously illustrated positive or negative impulse responses in the tumor ROI due to inhaling medical oxygen. Correlation coefficient mapping demonstrated heterogeneity in tumors after inhaling medical oxygen. BOLD and TOLD signals exhibited increased changes in signal intensities after the first fraction of dose. Multi-fractionation had minimum effect until the second

Presenting author: Derek A White; UT Arlington Biomedical Engineering, Texas, USA.

Cite this article as:

White DA, Mason RP. Non-invasive pre-clinical MR imaging of prostate tumor hypoxia for radiation therapy prognosis. Int $J$

Cancer Ther Oncol 2014; 2(2):020243.

DOI: $10.14319 /$ ijcto.0202.43 fraction of dose was applied. Tumor growth delays were observed when inhaling medical oxygen during IGRT.

Conclusion: OE-MRI is a non-invasive imaging modality that can provide insight to the oxygen status of tumors. Observed increase percent changes in BOLD and TOLD signal intensities after the first fraction of dose suggest tumors experienced reoxygenation. OE-MRI could be used for predicting tumor response to IGRT when using medical oxygen for increasing GTV radiosensitivity, suggesting patient stratification for clinical implementation.

Innovation/Impact: Oxygenation in tissue has been shown to influence MR physical parameters such as the intrinsic longitudinal relaxation time $\left(\mathrm{T}_{1}\right)$ and the apparent transverse relaxation time $\left(\mathrm{T}_{2}{ }^{*}\right)^{1-5}$. Investigations have shown that molecular oxygen when inhaled and dissolved in tissue fluid or blood plasma, can alter $\mathrm{T}_{1}$ values in the tissue due to its paramagnetic characteristics ${ }^{3,5}$. Similarly, alterations of $\mathrm{T}_{2}{ }^{*}$ values can be observed in the blood due to the magnetic susceptibility differences between oxy- and deoxyhemoglobin ${ }^{3}$. Tumor hypoxia is associated with radiation resistance. However, hitherto there is no satisfactory method to accurately determine the distribution of partial pressure of oxygen $\left(\mathrm{pO}_{2}\right)$ in tumors non-invasively. It has been suggested that Oxygen-Enhanced MRI (OE-MRI) may provide insights into tumor hypoxia. I am exploring a non-invasive imaging technique (Interleaved BOLD/TOLD - IBT) for determining the state of tumor oxygenation based on changes in signal intensity from the dependency of BOLD or $\mathrm{T}_{2}{ }^{*}$ (blood-oxygen level dependent) and TOLD or $\mathrm{T}_{1}$ (tissue-oxygen level dependent) response to a hyperoxic gas challenge prior 
to irradiation, as potential prognostic biomarkers of response. Indeed, the predictive value may depend on tumor type and thus it is crucial to evaluate these

Key Results: For this radiobiological study, experimental tumor models included an adult male nude rat implanted with $2.5 \times 10^{6}$ PC-3-luc human prostate cancer cells in the right flank and Dunning R3327-AT1 prostate tumors (typical doubling time approximately 5 days) growing subcutaneously in syngeneic Copenhagen rats. The size of experimental tumor models in- methods in diverse tumor types and sites of implantation.

cluded small (1 cc) and large volume tumors (2.5 cc). For AT1 prostate tumors, tumor growth delay (TGD) was determined by tumor measurements for up over 40 days or until reached 2 - 4 times the irradiated tumor volume. For proof-of-principal, the PC-3 prostate TGD was determined by tumor measurements up to 200 days. Figure 1 and 2 are from a representative large ATI tumor.

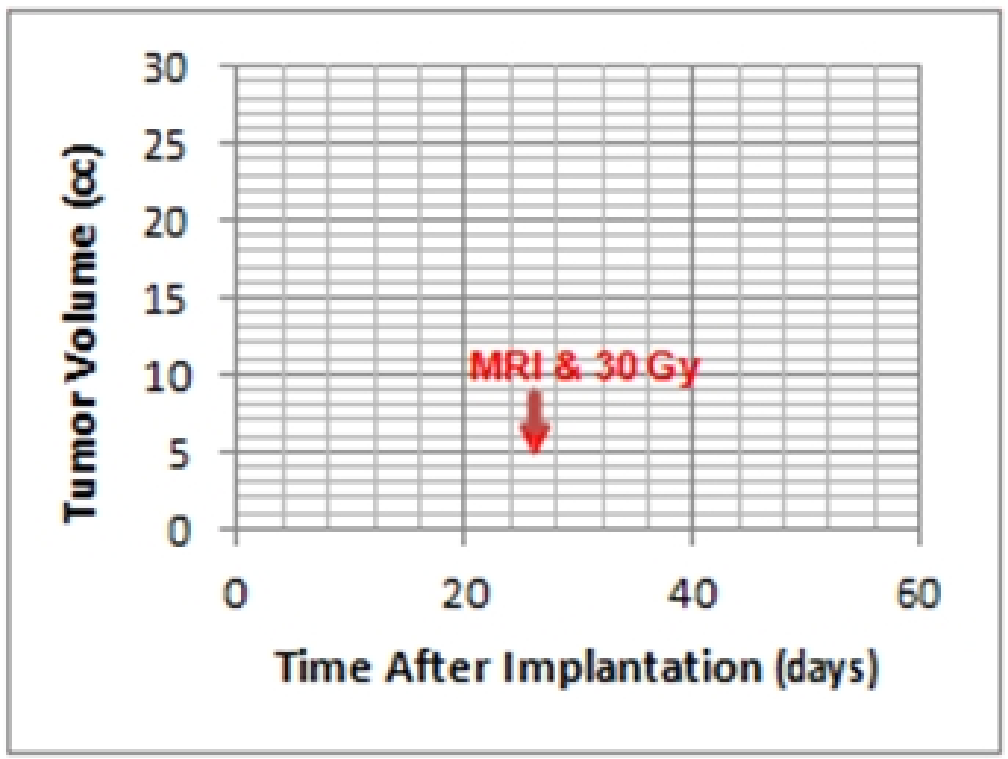

FIG. 1: Tumor Growth

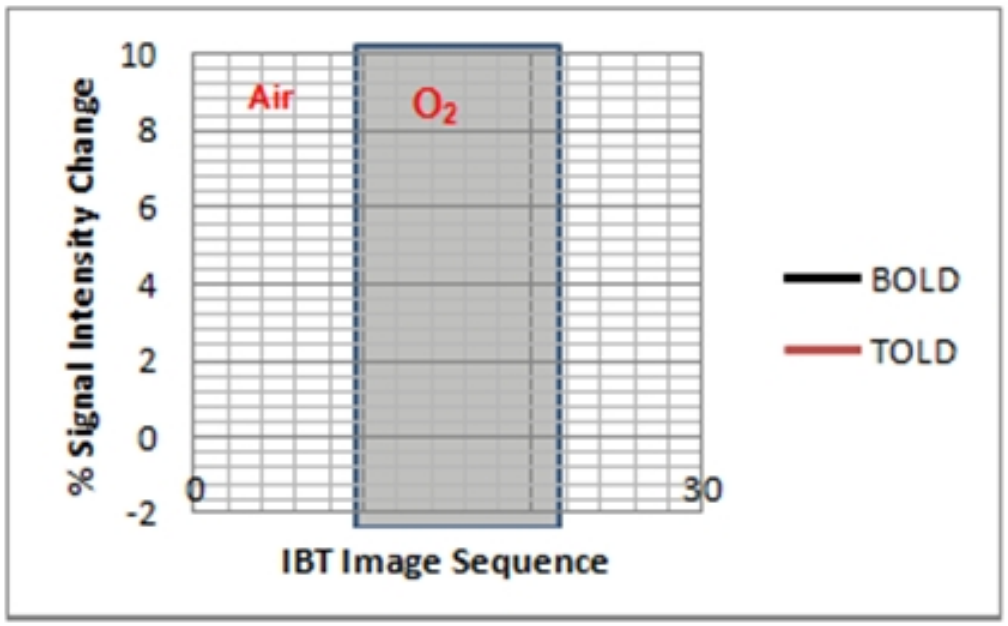

FIG. 2: Dynamic OE-MRI

\section{References}

1. Zhang Z, Hallac RR, Peschke P, Mason RP. A noninvasive tumor oxygenation imaging strategy using magnetic resonance imaging of endogenous blood and tissue water. Magn Reson Med 2014; 71: 561-9. 
2. Hallac RR, Zhou H, Pidikiti R, Song K, Stojadinovic S, Zhao D, Solberg T, Peschke P, Mason RP. Correlations of noninvasive BOLD and TOLD MRI with $\mathrm{pO}(2)$ and relevance to tumor radiation response. Magn Reson Med. 2013.[Epub ahead of print]

3. Huen I, Morris DM, Wright C, et al. R1 and R2 * changes in the human placenta in response to maternal oxygen challenge. Magn Reson Med 2013; 70: 1427-33.

4. Burrell JS, Walker-Samuel S, Baker LC, et al. Exploring $\Delta \mathrm{R} 2^{*}$ and $\Delta \mathrm{R} 1$ as imaging biomarkers of tumor oxygenation. $J$ Magn Reson Imaging 2013; 38: 429-34.

5. O'Connor JP, Naish JH, Jackson A, et al. Comparison of normal tissue R1 and R*2 modulation by oxygen and carbogen. Magn Reson Med 2009; 61: 75-83. 